\title{
Semantically-enhanced Configurability in State Estimation Structures of Power Systems
}

\author{
Georgios M. Milis, Markos Asprou, Elias Kyriakides, Christos G. Panayiotou, and Marios M. Polycarpou \\ KIOS Research Center for Intelligent Systems and Networks \\ Department of Electrical and Computer Engineering \\ University of Cyprus \\ Nicosia, Cyprus \\ Email: \{milis.georgios, asprou.markos, elias, christosp, mpolycar\}@ucy.ac.cy
}

\begin{abstract}
The estimation of the states of an electric power system, that is, the magnitude and angle of the voltage at all buses, is a very critical input to many monitoring and control functions of power systems. The recently witnessed rapid deployment of synchronized measurement technology (SMT) in power systems, has led to research advancements in the state estimation technology that introduce the notion of hybrid state estimation. These techniques incorporate the synchrophasors provided by the Phasor Measurement Units (PMUs) in the state estimation process, thus improving the state estimation accuracy. However, both the traditional as well as the hybrid techniques, assume a pre-defined configuration and characteristics of the measurement devices. This work explores how semantic modelling and reasoning techniques may contribute to the online configuration of the state estimation architectures given the available measurement capabilities at each moment.
\end{abstract}

\section{INTRODUCTION}

The Electric Power System (EPS) comprises a very critical infrastructure for the operation of our modern society and economy. Therefore, the effective monitoring and control of an EPS is considered of utmost importance and is undertaken by a set of components that perform a wide range of functionalities and together comprise the "Energy Control Centre" (ECC) application. Considerable resources are allocated by international organizations and governments, as well as private organisations, to advance the state-of-art and subsequently the effectiveness of all components of the ECC.

\section{A. State Estimation Architectures}

One of the most critical components of an ECC, is the "State Estimator" (SE), which serves several functions that support the reporting to the human operators, as well as functions that support the operation planning, the stability and the security of the EPS. The key position of the SE is illustrated in Fig. 1 [1]. In essence, the SE produces an estimate of the operating state of the system (i.e., voltage magnitude and phase angle of each bus) in consecutive time intervals, by processing redundant measurements acquired by selected substations of the system. The usual measurements are the real and reactive power injections, real and reactive power flows of the transmission lines, and the voltage magnitudes at the system buses. A strict prerequisite for obtaining a unique solution by the state estimator is to have a fully observable power system by the measurements used in the SE.

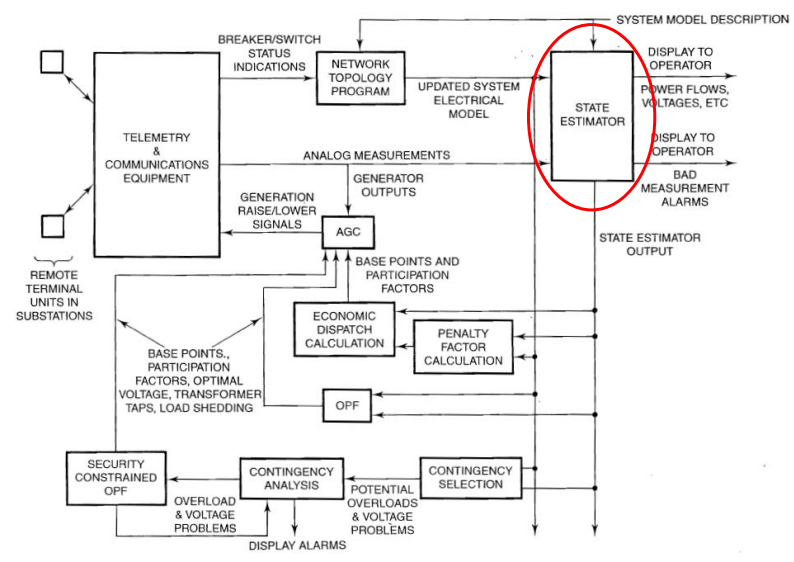

Fig. 1: The typical architecture of an ECC, where the key position of the SE is highlighted

The reliance of many ECC components on the state estimation imposes that the SE must provide as accurate and reliable results as possible. With the recent advancements in the measurement technology of EPS and the observed progress in the actual deployment of Phasor Measurement Units (PMUs) in the measurement layer of EPS, the research community has been investigating ways to take advantage of the available synchronized phasor measurements for improving the performance of the SE. Although the cost of PMUs has been decreased and is foreseen to further decrease in the near future, at the moment the measuring and communication infrastructure required for the deployment of PMUs turn the adoption of SE that rely solely on the PMU measurements impractical. This is further emphasized by the fact that conventional measurements can be useful in many other monitoring and control functions, such as measurement calibration and bad data detection methods [2]. Therefore, the research has been focusing on hybrid architectures for the SE that utilise both conventional and synchronized measurements for estimating the operating state of the EPS. A potential problem in the hybrid architectures is the inclusion of the current phasor measurements (PMU measurements) in the measurement vector that is usually detrimental to the performance of SE [2]. Many alternative techniques have been proposed for overcoming this issue, by utilising the concept of "pseudo measurements" [3][7]. , converting the current measurement to e.g., active power 
flow through an appropriate transformation function.

\section{B. The need for flexible architectures}

In practice, the measurements from the EPS are retrieved by physical devices of appropriate types and of a variety of (vendor-dependent) specifications. Therefore, either the SE algorithm developer needs to design the algorithm based on pre-acquired knowledge about the available devices and their specifications or the technicians that install the devices need to know the specifications of the SCADA system (mainly the SE implementation) in advance. These cases show the existing inflexibility of the current architecture of the SE.

The today's Smart Grid advancements [8] with the improved integration of the Electric Power Grid with the ICT infrastructure, open up additional opportunities to offer flexibility in the SE architectures. Given the fact that the measurement capabilities may vary spatially and temporally, as well as the fact that different SE algorithms may be required depending on the available measurement capabilities, this work contributes with the introduction of an SE architecture that allows online configurability and can be employed in several ECC applications. The proposed SE architecture is enriched with a semantic composition layer, which stores structured knowledge about the available components (e.g., measurement devices and SE algorithms' implementations), performs semantic reasoning after any change in the available components and takes decisions about the online re-configuration of the SE. The situation awareness is achieved by utilising a predesigned domain ontology that fully describes the types of the components, their characteristics, their locations, as well as the physical properties they measure.

The paper is organised as follows: Section II formulates the challenge, Section III presents the proposed architecture and methodology, followed by Section IV where the knowledge storing and reasoning mechanism is presented. Then, Section $\mathrm{V}$ presents a case-study to facilitate the understanding of the proposed method, and finally Section VI concludes the paper and discusses future directions.

\section{Challenge Formulation}

The common denominator in all hybrid state estimation techniques is that they require the use of a different SE implementation to be able to consume both types of measurements under synchronisation conditions. In current practice, the design of SE architectures is based on a fixed configuration of specific sub-components, with static measurement devices configuration and predetermined state estimation routines. That is, the EPS operators need to decide in advance whether they will perform the state estimation based only on conventional measurements or whether they will adopt any of the hybrid architectures discussed above, together with the corresponding (static) implementation of the SE. Although this does not create significant inconvenience in today's operation procedures, since the need for changes in the components (i.e., sensing devices and SE implementations) is not so frequent, it does comprise lack of flexibility for the SE component. The flexibility may become more important as the interdependency of EPS with ICT infrastructure is increased. A "smart" implementation would be expected to present online adaptability to changes in the composition of the measurement vector (e.g., introduction of new conventional and/or PMU devices, removal or moving of PMU devices to other locations of the network topology, availability of new SE implementations) and perform any necessary re-configurations, in order to avoid the need of manual replacement of components and subsequent downtime.

A solution to this problem would be the introduction of an intermediate layer, which would be aware of any new situation, have at its disposal a set of components with their own characteristics and input/output mappings structurally described and be capable of taking informed online decisions on how to implement the SE. Such mediation architectures have been proposed for the automatic composition of web services by the Internet community. The composition is achieved using standard ontology frameworks that allow semantic composition of services/components, such as OWL-S [9] or frameworks for semantic annotation of RESTfull services [10]. Recent efforts in the framework of the "Internet of Things" paradigm, promote the use of these technologies in a cyber-physical perspective, addressing the additional spatio-temporal challenges involved with the interaction with the physical world. Therefore, ontologies have been proposed, dedicated to sensor annotations such as the "SSN" [11] and the "SensorML" standard [12]. Individual components are then semantically described using the pre-defined frameworks in combination with domain ontologies, which allows the selection of the appropriate components in a composition aiming to achieve a more complex objective.

\section{A. Current SE Techniques and Architectures in Power Systems}

The state estimation process can be illustrated with the block diagram of Fig. 2, where the vector of the states of the EPS (that is, the voltage magnitude and angle at all buses) is defined as $x \in \mathcal{R}^{n}$, the measurements' vector produced by the installed set of measurement devices $(\mathcal{S})$ is given by $y \in \mathcal{R}^{p}$, while $\hat{x} \in \mathcal{R}^{n}$ denotes the vector of the estimated system states. The diagram shows that the SE uses the set of available measurements as input and produces an estimate of the system states which is then utilised by several other monitoring and control functions that act on the system and support its operation.

The estimation of the EPS state is performed based on different approaches, with the one most commonly used being the WLS (Weighted Least Squares). According to the WLS method, the state vector $x$ of the system is determined iteratively by minimizing the weighted residuals between the estimated and the actual acquired measurements, $J(x)=[y-h(x)] W[y-h(x)]$, where $h(x)$ is the functionvector associating the state variables to the measurements, and $W$ is the inverse of the measurement error covariance matrix. Details on these state estimation algorithms can be found in [2]. It is assumed that the network topology and parameters are known prior to state estimation and also the EPS is completely observable by the measurements contained in vector $y$. In general, the measurement vector of an SE is given as $y=\left[P_{\text {flow }}, P_{\text {inj }}, Q_{\text {flow }}, Q_{\text {inj }},|V|, \theta_{V},|I|, \theta_{I}\right]^{T}$, where the elements represent respectively vectors of active and reactive power flows in lines, active and reactive power injected on buses, as well as voltage and current magnitudes and angles. In case of no availability of PMU units, the phasor 


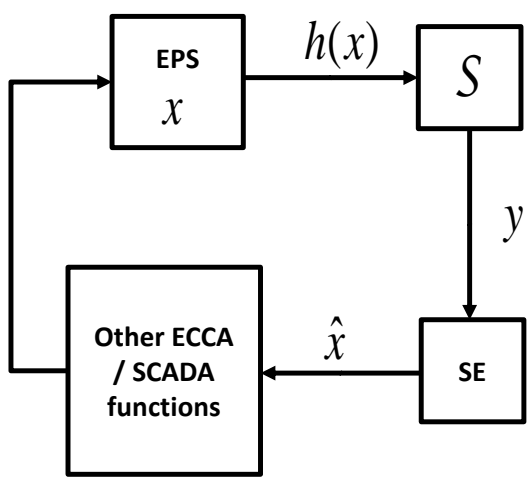

Fig. 2: Block diagram of the SE implementation, focusing on the relation with the plant, the measurement signals and the rest of the monitoring and control applications.

measurements are not present and the measurement vector is adjusted accordingly.

\section{B. Challenges phased by hybrid SE architectures}

It is emphasised here that, on one hand it has been proved beneficial for the SE to use a hybrid structure for exploiting also the PMU measurements [13], but on the other hand the PMU devices, like any measurement device, are subject to failures (e.g., broken GPS communication links) which would turn the hybrid SE not applicable and subsequently would necessitate the return to a conventional SE architecture. Furthermore, the current widely adopted practice by utilities, is to operate a SE that considers the locations of measurement devices pre-defined. However, the today's advancements in technology may justify a demand for additional flexibility in the SE architecture which would turn it possible to adapt online to changes happening in the content of the measurement vector, as well as, to changes in the available SE algorithms' implementations.

More specifically, assuming a conventional SE, operating on a vector of conventional measurements (no phasor measurements), it is desirable for the SE architecture to be able to adapt online when PMUs are installed in specific locations of system topology. It is also desirable for the SE to continue operating when these PMUs are mobile and can therefore move from one location to another, which will subsequently change the mapping to the system states. Finally, the SE should continue operating when PMUs or conventional devices are removed from the network due to any reason (assuming still an observable system). The following section presents the proposed architecture that offers a promising solution to the above described challenge.

\section{Methodology And Proposed Architecture}

The proposed architecture for the ECC, focusing on the SE part, is depicted in Fig. 3, where $I \in\{0,1,2, \ldots\}$ is the index of the SE architecture configuration. In configuration $I$, the operation of the EPS is monitored by a set of sensors $\mathcal{S}^{(I)}$, e.g., conventional sensors and PMUs. It has been explained earlier how in hybrid SE implementations, specific types of measurements (e.g. measurements of current) may degrade the SE performance. In such cases, the measurements pass through a set of appropriate transformation functions $\mathcal{F}^{(I)}$ before given to the selected $\mathrm{SE} \mathcal{S E} \mathcal{E}^{(I)}$.

In order to achieve the objective of shifting from configuration $I$ to $I+1$ when required, the use of a Semantic Mediation Agent $\Sigma$ is proposed, which is responsible for making the decisions and configuring all components. The agent $\Sigma$ first detects and identifies any new component(s) added. The physical communication among components is facilitated through an assumed existing communication protocol, e.g., with extensions to the currently adopted SCADA systems (the details of this fall outside the scope of this work). Subsequently, $\Sigma$ becomes aware of the characteristics and capabilities of the new components, which is exactly the emphasis of this work. The use of ontological knowledge models and semantic annotation and mediation techniques are proposed [14], [15]. Each component is assumed pre-annotated with certain "tags" that will describe its characteristics and capabilities. Once this information is received by $\Sigma$, it is stored and integrated with the existing knowledge about the overall system, depicted by the knowledge model $\Lambda$. It is noted that in future implementations of Smart Grid, it may become possible for information to be obtained by remote users and/or from the Internet. The given knowledge is in turn used to infer new (implicit) knowledge, denoted by $\Lambda_{m}$. Subsequently, the model is utilized to reconfigure the existing SE architecture considering all available components, e.g., to return to conventional SE if PMU measurements are lost. Every time a new measurement unit and/or SE implementation is added, agent $\Sigma$ detects and identifies it and its main task is to become aware of the new component's functionality, properties and characteristics by exploring also additional information from third party Internet services or human users. It is emphasised here that any shift from configuration $I$ to $I+1$ happens strictly in the time between subsequent estimation cycles and no interruption of a running estimation process is performed. It is assumed that the time between two subsequent estimation executions is enough to allow / Sigma to complete the reasoning and decide the new configuration.

The key advantage of the proposed architecture is the inherited flexibility and automation of the components' wiring layer, through the incorporation of a "semantic" layer. Structuring the knowledge representation, enables a machine (through the $\Sigma$ agent) to undertake tasks that would otherwise be undertaken by humans (e.g., the update of the SE implementation when the measurement vector changes). The same approach can be adopted in other parts of the ECC application, where such flexibility may be of use. On the other hand, the main drawback of the architecture is that it requires spending considerable effort offline, to create correct knowledge models and semantic annotations for the involved components. This is a trade-off that needs to be considered depending on the application and the estimated benefits. The following section gives details about the ontology-based knowledge model, as well as about the reasoning mechanism. 


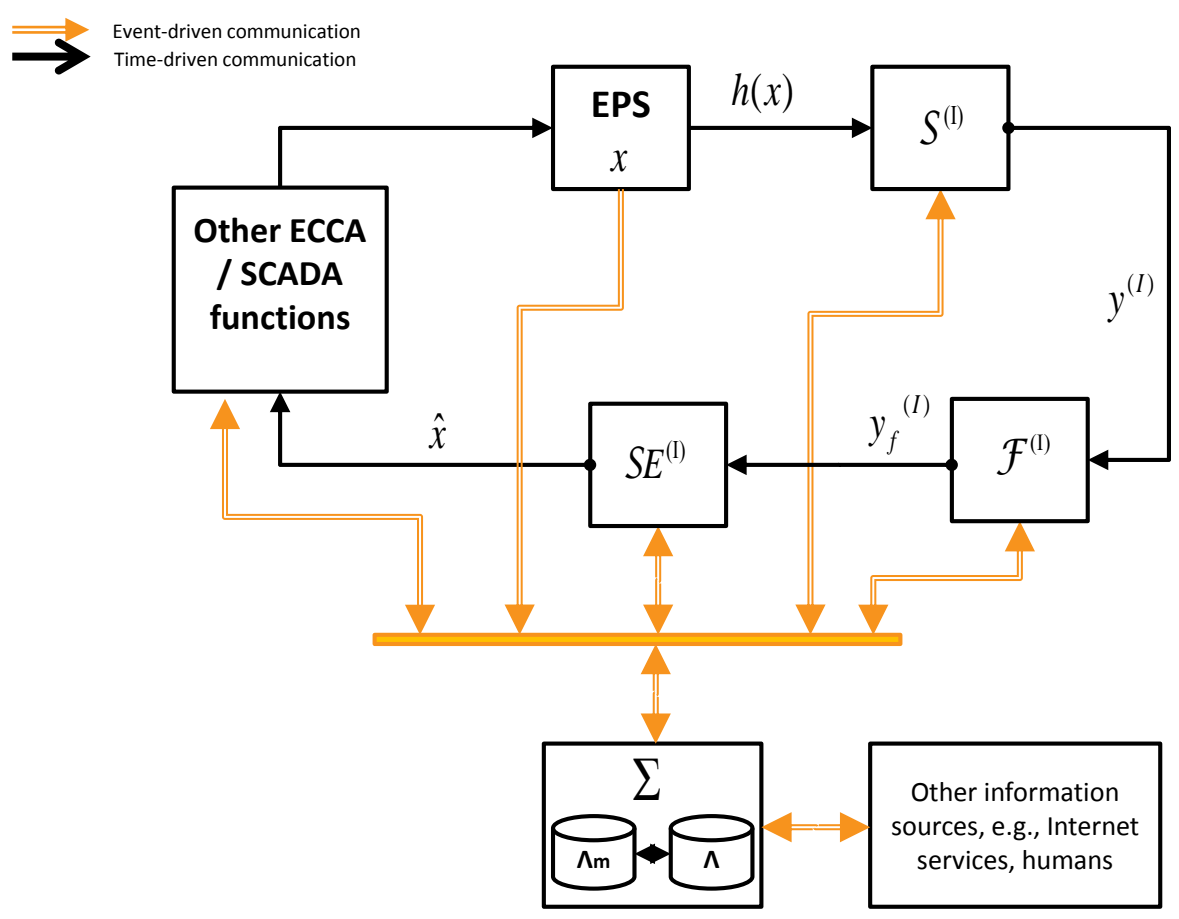

Fig. 3: Block diagram of the proposed SE architecture implementation, where the agent $\Sigma$ is introduced with event-driven communication and knowledge exchange capabilities, so as to reason about appropriate wiring of components. The diagram shows the set of used sensors $\mathcal{S}^{(I)}$, the set of used $\mathrm{SE} \mathcal{S} \mathcal{E}^{(I)}$ and the set of available transformation functions $\mathcal{F}^{(I)}$. The time-driven communication concerns vectors of signals each time with appropriate dimension. The event-driven communication comprises two-way cyber communication with components, to facilitate their semantic annotations' sharing, as well as the online wiring. Note that communication with the EPS is one-way since the system is assumed as not communicating anything directly to the agent $\Sigma$ (any knowledge about it comes from human or cyber sources).

\section{THE KNOWLEDGE MODEL AND SEMANTIC REASONING IMPLEMENTATION}

The knowledge model adopted in this work, is implemented as a light ontology to facilitate the presentation of the concept. This ontology will be merged in the future with existing standard ontology frameworks that allow semantic composition of services/components, as has been discussed in Section II. In general, knowledge models help in facing the interoperation issues by implementing structured representations of domain knowledge and by providing suitable reasoning facilities to make best possible use of the combined stored knowledge. The scope in this work is to achieve online SE components' composition and interoperability.

An illustrative case-study follows, aiming at clarifying how the knowledge model is built, as well as, how the logical (semantic) reasoning is performed over the stored knowledge facts to implement the decision mechanism for the online configuration/composition of the SE.

\section{A. Example System and Concepts}

Let assume a three-bus EPS as in Fig. 4, where five measurement devices are installed, performing twelve sensing

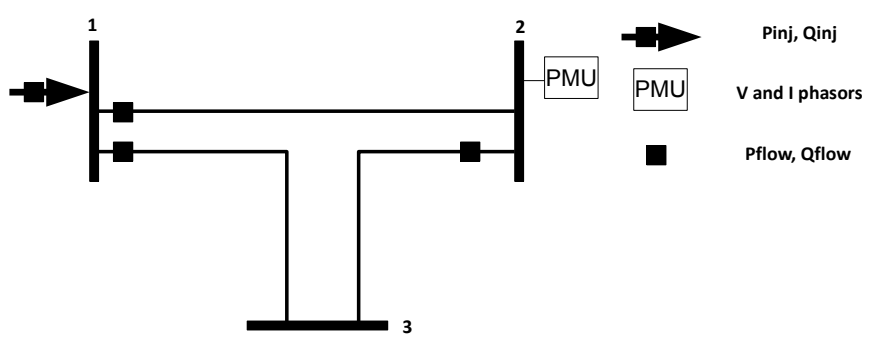

Fig. 4: Three-bus EPS with five measurement devices (12 single measurements)

tasks in total. As shown, the devices measure the injected power on bus 1 , the flow of power on lines $1-2,1-3$ and $2-3$, as well as the voltage and current phasors on bus 2 . The voltage magnitude is measured in $\mathrm{kV}$, the current magnitude in $\mathrm{kA}$, the angles in degrees and the power in $\mathrm{kW}$ (active) and kVAr (reactive).

The introduced system contains a number of "things". 
There are essentially twelve sensors $\left(s_{i}, i=1, \ldots, 12\right)$, the physical properties "active electric power" $\left(q_{1}\right)$, "reactive electric power" $\left(q_{2}\right)$, "voltage magnitude" $\left(q_{3}\right)$, "current magnitude" $\left(q_{4}\right)$, "voltage angle" $\left(q_{5}\right)$ and "current angle" $\left(q_{6}\right)$, the measurement units "kiloWatts" $\left(m_{1}\right)$, "kiloVArs" $\left(m_{2}\right)$, "KiloVolts" $\left(m_{3}\right)$, "kiloAmpers" $\left(m_{4}\right)$, "degrees" $\left(m_{5}\right)$, "per-unit" $\left(m_{6}\right)$, as well as the buses and transmission lines as system locations $\left(l_{j}, j=1, \ldots, 6\right)$. All these are either cyber-physical components or other types of linguistic representations which are modelled as knowledge objects (elements of a set $\mathcal{N}$ ) in the dedicated ontology. Moreover, the type-set of each object (e.g., "kiloWatts" is a measurement unit while "bus1" is a location) is also defined in the ontology. Therefore, the set of sensors $(\mathcal{S})$, the set of locations $(\mathcal{L})$, the set of physical properties $(\mathcal{Q})$, as well as the set of measurement units $(\mathcal{M})$ are defined. These are essentially elements of a types-set $\Omega$ and subsets of $\mathcal{N}$. Classical sets of objects, like the set of real numbers $(\mathcal{R})$, can also be used in the ontology. Each of these type-sets has a separate finite cardinality $n_{V}$, where $V$ is any element of $\Omega$.

In addition, the full understanding of the meaning is facilitated by the relations between the existing objects. That is, "kiloWatts" $\left(m_{1} \in \mathcal{M}\right)$ is a measurement unit of "active electric power" $\left(q_{1} \in \mathcal{Q}\right)$, while the sensor $s_{1}$ is located on "bus1" $\left(l_{1}\right)$. That is, the relations can be modelled as (nonbalanced) bipartite graphs where edges define mappings among vertices of a domain object-set to a range object-set. This definition of relation is given below:

Definition: $G\left(V^{o}, V^{d}, E^{\left(V^{o}, V^{d}\right)}\right)$ : defines a non-balanced bipartite graph (called here also relation graph) with vertices being the elements of the sets $V^{o}=\left\{v_{i}^{o} \mid i=1,2, \ldots, n_{V^{o}}\right\}$ and $V^{d}=\left\{v_{j}^{d} \mid j=1,2, \ldots, n_{V^{d}}\right\}, V^{o}, V^{d} \in \Omega$, and edges being the elements of the set $E^{\left(V^{o}, V^{d}\right)}=\left\{\left(v_{i}^{o}, v_{j}^{d}\right) \mid v_{i}^{o} \in V^{o}, v_{j}^{d} \in V^{d}\right\}$, which represent the connections between elements of the origin set $V^{o}$ to elements of the destination set $V^{d}$.

Examples of relations might be the relation between sensors and locations $G\left(\mathcal{S}, \mathcal{L}, E^{(\mathcal{S}, \mathcal{L})}\right)$, relation between sensors and measurement units $G\left(\mathcal{S}, \mathcal{M}, E^{\mathcal{S}, \mathcal{M}}\right)$, relation between measurement units and physical properties $G\left(\mathcal{M}, \mathcal{Q}, E^{(\mathcal{M}, \mathcal{Q})}\right)$, relation between location objects representing the case where a location "is part of" another location $G\left(\mathcal{L}, \mathcal{L}, E^{(\mathcal{L}, \mathcal{L})}\right)$ and relation between sensors and real numbers $G\left(\mathcal{S}, \mathcal{R}, E^{(\mathcal{S}, \mathcal{R})}\right)$ to represent the accuracy of a measurement. Graphical representations follow later in the paper to clarify the meaning of relations.

The ontology-based knowledge model, in fact, comprises the convention/agreement between all physical and soft/cyber components that interact in the SE implementation, about the interpretation of their capabilities (e.g., inputs/outputs) and the domain in which they operate. The description of a new object using the pre-defined ontology is called "semantic annotation of the object". For instance, the semantic annotation of the sensors in this work comprises information about the location of the corresponding device, the units of the produced values (e.g., kiloWatts, kiloVolts, kiloAmper) and the accuracy of the produced values as given by the manufacturer. Then, the knowledge model is exploited by the $\Sigma$ component to take rational decisions about which measurements, which SE algorithm implementations, as well as what other transformation functions to use and how to organise these components in the composition of the SE architecture. The logical decisions are taken based on inference rules, written using the SPARQL Protocol and the RDF Query Language, [16].

The following subsection shows how the components are modelled (annotated) in terms of the defined knowledge model.

\section{B. Semantic modelling of SE Components}

What is achieved with semantic annotation is the encoding of the required knowledge in machine readable format, while the objective is to use this knowledge in order to determine the components (cyber and physical) to be used towards synthesizing the SE architecture. In order to better understand the semantic compose-ability of components, their semantic annotations are graphically introduced here. Since the connection of components is achieved through their inputs and outputs, the adopted convention is for the components to be first described (annotated) in terms of their inputs and outputs (sets $\mathcal{T}$ and $\mathcal{O}$ respectively), assuming the inputs and outputs properly inherit the semantic characteristics of the components.

Fig. 5 illustrates the semantic annotation of a sensor $s_{i}, i=1, \ldots, n_{\mathcal{S}}$ with one output $o_{i}, i=1, \ldots, n_{\mathcal{O}}$ (the modelling of sensor inputs from the physical signals of the power system are out of the scope of this work). The sensor and its output shown in the "Implementation Layer", are also represented by knowledge objects in the "Knowledge Model Layer". Then the output is associated with some measurement unit at some location (bus or line) of the EPS (accuracy is omitted here as it is not used in the reasoning algorithm). The knowledge objects are shown with circles, while the sets to which they belong are shown with dashed-line rectangle containers. Subscripts $i, j \in\left\{1, \ldots, n_{V}\right\}, V \in \Omega$ are used to demonstrate the potential existence of many objects of same type and differentiate between them. The relations between the objects are shown by the edges (thick-black or thingrey depending on whether the edge is part of the object's semantic annotation or not). For further clarity, the knowledge model is split into four layers: i) the "System Components" layer which contains the knowledge objects that represent the actual implementations of components, ii) the "Inputs/Outputs" layer which contains all inputs and outputs of components, iii) the "Thematic Knowledge" layer which contains all other domain specific knowledge objects that are used to annotate the components, and iv) the "Functions" layer which hosts knowledge objects representing functions that e.g., transform one measurement unit to another.

In the case of a state estimator, the reasoning performed in this work only uses its inputs $\left(t_{i}, i=1, \ldots, n_{\mathcal{T}}\right)$, therefore output annotation is omitted. The semantic annotation model of a SE is shown in Fig. 6. It is assumed that the topology of the system is known to the SE implementation and that it is able to retrieve the pre-defined mappings of measurements to states and thus build the $h(x)$ function online.

Additional inputs of SE and outputs of sensors are modelled in the same way, with their corresponding annotations.

\section{Semantic reasoning mechanism}

Let assume now a SE architecture as in Fig. 3, deployed in the control centre of the introduced EPS, such as to estimate 


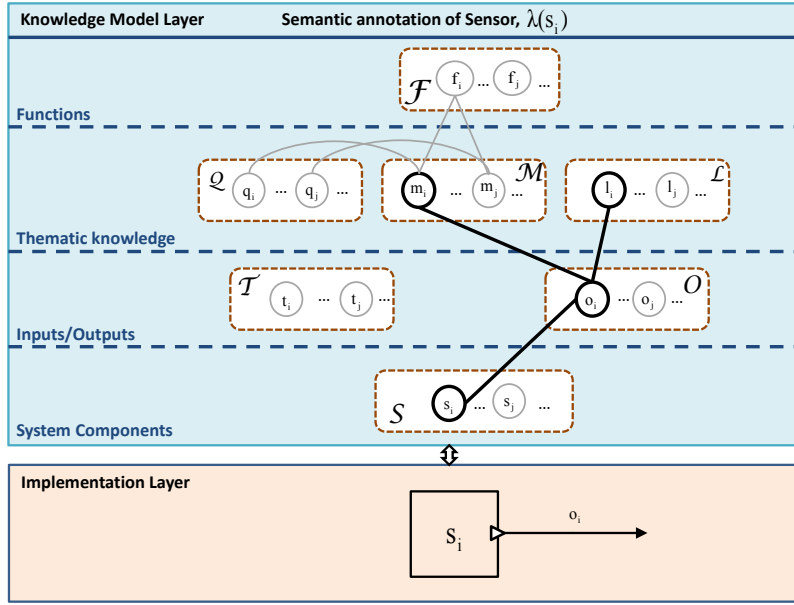

Fig. 5: The semantic annotation model of a "sensor" $s_{i}, i=$ $1, \ldots, n_{\mathcal{S}}$ with one output $o_{i}, i=1, \ldots, n_{\mathcal{O}}$

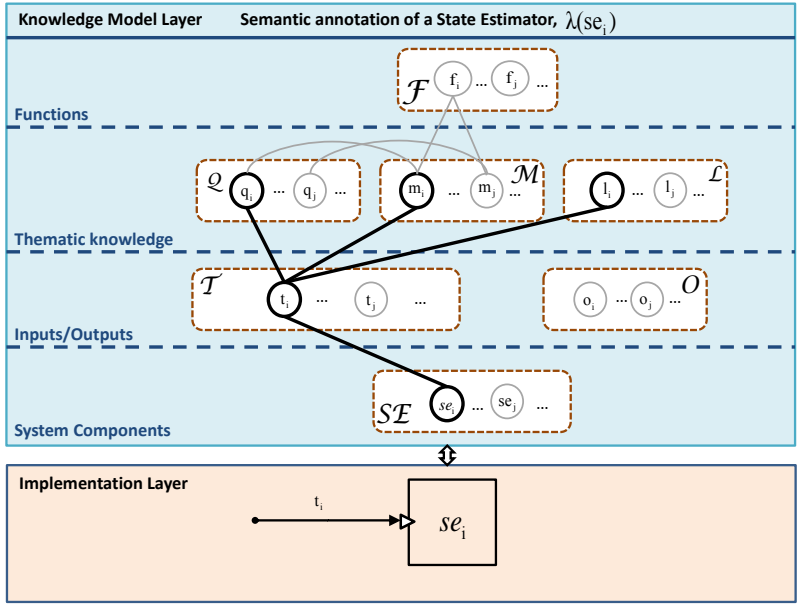

Fig. 6: The semantic annotation model of a "SE" $s e_{i}, i=$ $1, \ldots, n_{\mathcal{S E}}$ with one input $t_{i}, i=1, \ldots, n_{\mathcal{T}}$

the voltage magnitudes and angles of the three buses. The mapping of the measurement vector to the system states is given by (1),

$$
y_{f}^{(1)}=f\left(y^{(1)}\right)=f\left(h(x)^{(1)}+w^{(1)}\right)
$$

where $x \in \mathcal{R}^{5}$ : The voltage magnitudes and angles of the three buses, in per-unit (pu).

$y^{(1)} \in \mathcal{R}^{12}:$ The measurements (respectively sensors) produced by the deployed measurement devices.

$h(x)^{(1)}$ : Known mappings of system states to measurements. $w^{(1)}$ : The noise associated with the measurements

$f($.$) : A function-vector with elements f_{i} \in \mathcal{F}^{(1)}, i=1, \ldots, 12$ that are applied (if and when required) to the measurements, to ensure compatibility with what the SE expects to receive.

As explained, the objective is for the agent $\Sigma$ to choose a subset of components from the available set after a change event, based on their semantic composability, so as to ensure the SE operates properly. The components' composition algorithm first considers the types of the components and their expected role in the SE implementation. The position of each type of component is fixed in this work, with sensors always positioned to measure EPS outputs and then passing the measurements to the SE either directly or after processing. Then, the algorithm considers the matching of the inputs to the outputs based on their semantic properties. For instance, the location and the physical property (and/or the measurement unit) comprise important information about components' inputs/outputs. That is, the value produced by a sensor can be fed to the SE only if its location, physical property and measurement unit match to the respective properties expected by the SE. The available SE implementations are ranked offline (future work will try to address the automation of the ranking as well) based on the following criteria: i) give preference to hybrid implementations if PMUs exist, otherwise use conventional implementations, ii) give preference to implementations that make use of the maximum number of measurements. Therefore, in case more than one SE implementations satisfy the semantic matching, the one with higher ranking will be selected.

The querying within the knowledge model and the reasoning are facilitated in this work by SPARQL [16]. A semantic matching between an output and an input is confirmed only if all adjacent nodes of the input-node at the "Thematic knowledge" layer are reachable by paths starting from the output-node. The adopted "Components' Composition Decision Algorithm" is summarised as:

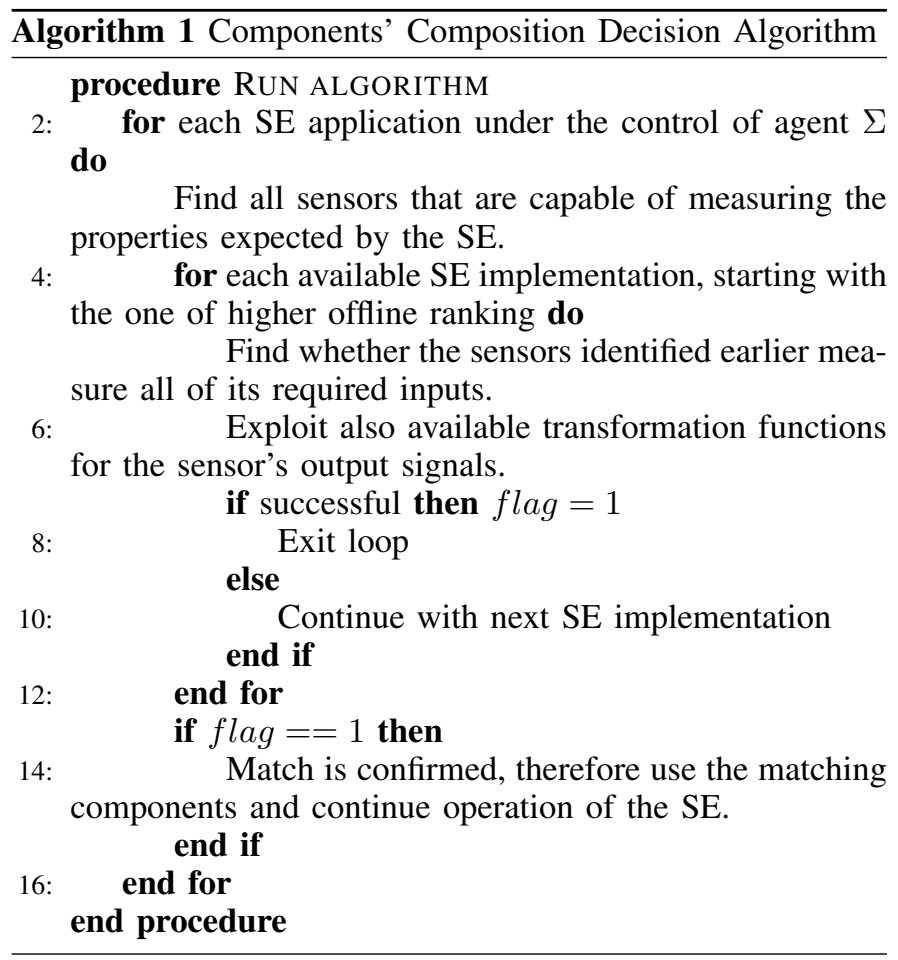

The execution of the algorithm will be made clearer with the illustrative cases in Section V.

\section{ILlUSTRATIVE CASE-STUDY}

For the purpose of presenting the concept, two SE implementations are assumed available $\left(s e_{1}, s e_{2}\right)$ in a tools' 
database, from which the agent $\Sigma$ will select according to the semantic matching output. The $s e_{1}$ is a WLS implementation of a conventional estimator and as such it uses only the eight conventional measurements from the twelve in the example EPS of Fig. 4. On each execution it (iteratively) estimates the EPS states. On the other hand, the $s e_{2}$ is a WLS implementation of a hybrid estimator, which is able to use additionally the synchrophasor measurements from the PMUs and integrate them in the iterative estimation of the states. The implementation is making use of "pseudo-measurements" to overcome the issue with the current phasor measurement, as in [6].

Concerning the measurement devices, it is assumed that no PMU is initially deployed at the EPS of Fig. 4. That is, the measurement vector at configuration $I=0$, comprises eight measurements (modelled here as eight sensors respectively), including the active and reactive power injected on Bus 1, as well as the active and reactive power flows of all three lines. As explained in Section IV-B, each of the measurements corresponds to a specific location in the EPS (e.g., specific bus or line side) and a specific measurement unit (e.g., $k W, V)$. On the other hand, the SE implementations need to receive measurements from specific locations, specific physical properties (e.g., Reactive Power, Voltage) and specific measurement units $(p u)$, as derived by the function vector $h(x)$.

Fig. 7 illustrates a part of the execution of the "Components' Composition Decision Algorithm", where on line 3 the semantic matching is checked between a single sensor $\left(s_{1}\right)$ producing a value in $k W$ at Bus 1 (measurement unit $m_{1}$ at location $l_{1}$ ) and one of the inputs of the SE implementation $s e_{1}$ representing the active power (physical property $q_{1}$ ) injected at Bus 1 (location $l_{1}$ ) in $p u$ (measurement unit $m_{2}$ ). The rest of the sensors and SE inputs are omitted here simply to hide complexity. It can be seen that all three nodes adjacent to the input $t_{1}$ of $s e_{1}$ in the "Thematic knowledge" layer, that is, nodes $l_{1}, q_{1}$ and $m_{2}$ marked with double black lines, are reachable by paths departing from output $o_{1}$ of sensor $s_{1}$, marked with single thick black line. Note that the matching is only possible through the transformation function $f_{p u}$ which is assumed appropriately transforming the $k W$ unit to $p u$. Matching of other measurements to respective inputs of the $s e_{1}$ is achieved in the same way. Therefore, at configuration $I=0$, considering the criteria for the offline ranking, the agent $\Sigma$ will select $s e_{1}$ to close the wire and execute the iterative state estimation.

Sometime in the future, the PMU is deployed on bus 2 (location $l_{2}$ ). This PMU is modelled with four (virtual) sensors in the knowledge model, measuring the magnitudes and angles of the voltage and current at the subject location. This change is detected by agent $\Sigma$ (details of detection are out of the scope of this work) and before the next state estimation execution, it runs the algorithm to check whether a new configuration $I=1$ can be achieved by utilising the PMU measurement as well. Checking for $s e_{2}$ first, which has the higher ranking if all measurements can be consumed, $\Sigma$ is able to confirm matching of all but one measurements to corresponding inputs of $s e_{2}$ (assuming the function-vector $h(x)$ of the $s e_{2}$ is updated properly). The not matching measurement is the current phasor assumed as produced by sensor $s_{2}$ in $k A$ (measurement unit $m_{3}$ ), which is not consumable by the specific hybrid SE

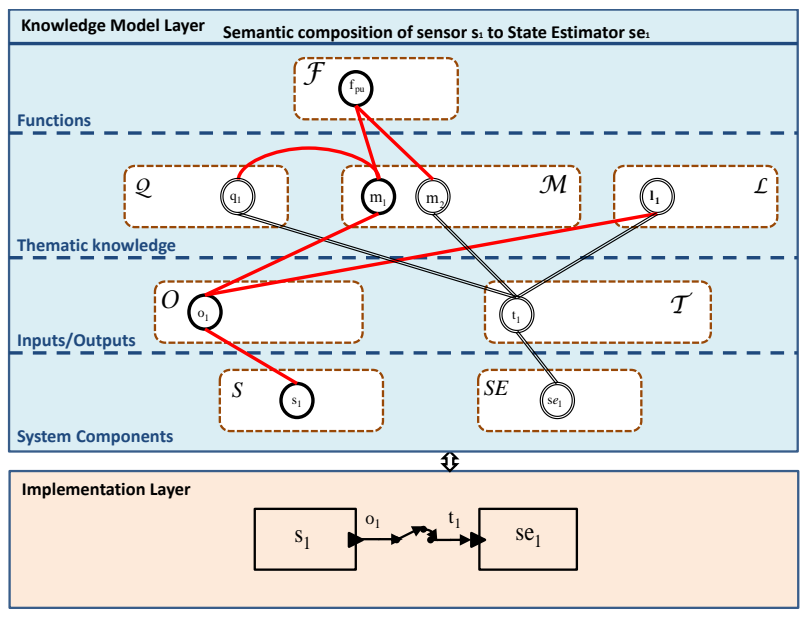

Fig. 7: The semantic matching of output $o_{1}$ of sensor $s_{1}$ to the input $t_{1}$ of SE implementation $s e_{1}$

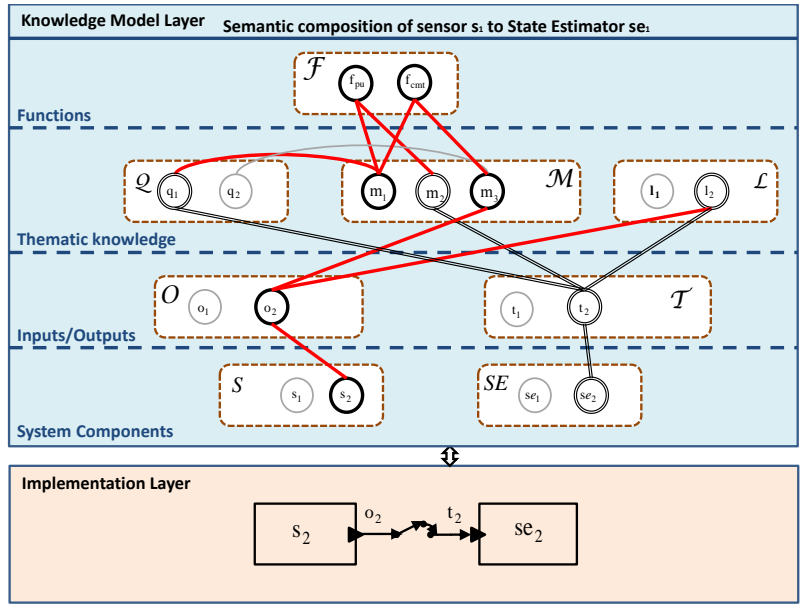

Fig. 8: The semantic matching of output $o_{2}$ of sensor $s_{2}$ to the input $t_{2}$ of SE implementation $s e_{2}$

implementation of $s e_{2}$, since it expects a power flow "pseudomeasurement" in $p u$. However, it is assumed that in parallel to the installation of the PMU, a function $f_{c m t}$ is imported in the tools' database and annotated accordingly in the knowledge model, which transforms the current measurement to the expected active power "pseudo-measurement". The resulted semantic matching is shown in Fig. 8. It can be seen that the location annotation matches directly, while the physical property annotation matches through the measurement unit $m_{3}$ which represents the $k A$ that is a measurement unit of current (physical property $q_{2}$ ). Then, the measurement unit annotation requires the path to pass through both transformation functions, $f_{p u}$ and $f_{c m t}$, to first transform kilo-amperes $\left(m_{3}\right)$ to kilowatts $\left(m_{1}\right)$ and then transform that one to $p u$ as required by the SE implementation. Therefore, the agent $\Sigma$ selects the hybrid estimator $s e_{2}$ and feeds its inputs with all available measurements in order to execute the state estimation. The $s e_{1}$ could also be used, however, it would not have used all available measurements, therefore, it is rejected from the predefined criteria. 
It is emphasised that the proposed architecture does not impose any limits to the complexity of the modelled thematic knowledge and reasoning mechanisms to be adopted. The examples presented here are kept simple for presentation purposes.

\section{CONCLUSIONS}

A flexible architecture has been described, that can be adopted in the design of new generation state estimation of power systems, in order to take advantage of the online reconfigurability characteristics offered by the semantic interoperability and composition techniques. The scope of the work was neither to advance the theory behind state estimation nor to advance the theory of semantic composition. It was rather to develop a mechanism that will allow the application of semantic composition and interoperability techniques within the SE function in an EPS. The current industrial practice suggests using standard SEs. We believe that recent advancements in SE algorithms, in combination with recent advancements of Smart Grid technologies, can benefit the utilities if there is a framework for their online deployment. In future steps, the flexibility of the architecture will be further improved by adopting standard ontologies. In addition, the semantic composition techniques will be extended to other parts of the power system control application, i.e. applications on the distribution grid that are usually more demanding in terms of flexibility and reconfigurability.

Acknowledgment: This work is partially funded by the European Research Council (ERC) under the project "FaultAdaptive Monitoring and Control of Complex Distributed Dynamical Systems".

\section{REFERENCES}

[1] A. J. Wood, B. F. Wollenberg, and G. B. Sheble, Power Generation, Operation and Control, 3rd ed. Wiley, 2013. [Online]. Available: http://eu.wiley.com/WileyCDA/WileyTitle/productCd-0471790559.html

[2] A. Abur and A. G. Exposito, Power system state estimation: Theory and Implementation, New York: Basel, 2004.
[3] S. Chakrabarti, E. Kyriakides, G. Valverde, and V. Terzija, "State estimation including synchronized measurements," in Power Tech Conference, Bucharest, 2009, pp. 1-5.

[4] T. S. Bi, X. H. Qin, and Q. X. Yang, "A novel hybrid state estimator for including synchronized phasor measurements," Electric Power Systems Research, vol. 78, no. 8, pp. 2452-2458, 2009.

[5] Z. Ming, V. A. Centeno, J. S. Thorp, and A. G. Phadke, "An alternative for including phasor measurements in state estimators," IEEE Transactions on Power Systems, vol. 21, no. 4, pp. 1930-1937, 2006.

[6] M. Asprou and E. Kyriakides, "Enhancement of hybrid state estimation using pseudo flow measurements," in Power and Energy Society General Meeting, 2011 IEEE, Detroit, USA, 2011, pp. 1-7.

[7] S. Chakrabarti, E. Kyriakides, G. Ledwich, and A. Ghosh, "On the inclusion of phasor measurements in a power state estimation," IET Generation, Transmission, and Distribution, vol. 4, no. 10, pp. 1104$1115,2010$.

[8] IEEE SmartGrid, "Smart Grid Experts, Information, News and Conferences." [Online]. Available: http://smartgrid.ieee.org/

[9] D. Martin, M. Burstein, J. Hobbs, O. Lassila, D. McDermott, S. McIlraith, S. Narayanan, M. Paolucci, B. Parsia, T. Payne, E. Sirin, N. Srinivasan, and K. Sycara, "OWL-S: Semantic Markup for Web Services," 2008. [Online]. Available: http://www.ai.sri.com/daml/services/owl-s/1.2/overview/

[10] J. Davis and M. S. Rajasree, "RESTDoc: Describe, Discover and Compose RESTful Semantic Web Services using Annotated Documentations," International journal of Web \& Semantic Technology, vol. 4, no. 1, pp. 37-49, Jan. 2013. [Online]. Available: http://www.airccse.org/journal/ijwest/papers/4113ijwest03.pdf

[11] A. Sheth, C. Henson, and S. Sahoo, "Semantic Sensor Web," IEEE Internet Computing, vol. 12, no. 4, pp. 78-83, Jul. 2008. [Online]. Available: http://knoesis.org/library/publications/SHS08ICColumn-SSW.pdf

[12] "Sensor Model Language (SensorML)." [Online]. Available: http://www.opengeospatial.org/standards/sensorml

[13] A. G. Phadke and J. S. Thorp, Synchronized phasor measurements and their applications. New York: Springer, 2008.

[14] G. Antoniou and F. V. Harmelen, "Web ontology language: Owl," in Handbook on Ontologies in Information Systems. Springer, 2003, pp. 67-92. [Online]. Available: http://link.springer.com/chapter/10.1007/978-3-540-92673-3_4

[15] G. M. Milis, C. G. Panayiotou, and M. M. Polycarpou, "Towards a Semantically Enhanced Control Architecture," in IEEE Multi-Conference on Systems and Control, Dubrovnik, Croatia, 2012.

[16] W3C, "Semantic Web Query Standards," 2004. [Online]. Available: http://www.w3.org/standards/semanticweb/query 